i-DUST 2010, $01002(2011)$

DOI: 10.1051 idust/201101002

(C) Owned by the authors, published by EDP Sciences, 2011

\title{
GPR imaging of a fracture zone in the Vaucluse Karst Aquifer using 2D eikonal inversion
}

\author{
D. Van Vorst ${ }^{1, a}$, M. Yedlin ${ }^{1}$, Y. Guglielmi ${ }^{2}$, F. Cappa $^{3}$, S. Gaffet ${ }^{3}$, M. Maxwell ${ }^{4}$, \\ and D. Oldenburg ${ }^{1}$
}

${ }^{1}$ University of British Columbia, BC, Canada

${ }^{2}$ Géologie des Systèmes Carbonatés, Université de Provence Aix-Marseille, Marseille, France

${ }^{3}$ Géosciences Azur, UNS/CNRS/OCA, Valbonne, France

${ }^{4}$ Golder Associates, Burnaby, BC, Canada

\begin{abstract}
A 2D travel-time inversion algorithm that uses a recently developed fast-sweeping eikonal solver is presented. The algorithm is fast, extremely easy to implement, and robust. It is applied to borehole radar data collected in the karst aquifer at LSBB (Laboratoire Souterrain à Bas Bruit) and the recovered permittivity profile is shown to be consistent with borehole televiewer and material measurements.
\end{abstract}

\section{INTRODUCTION}

The use of ground penetrating radar (GPR) in the determination of fluid distribution in a given lithology has been employed extensively in the last fifteen years. Reference [1] estimated the spatial correlation of hydrogeological properties (permeability) using synthetic GPR tomographic data generated for a crossborehole configuration and permeability values obtained from a single core. The core-scale permeability was related to the core-scale dielectric constant via a simple logarithmic relation. This provided the mapping required to convert the dielectric constant to the equivalent permeability. Reference [2] place the GPR data collection in a broader context by reviewing selected geophysical techniques, such as GPR, as applied to hydrogeological parameter estimation. A more complete review was given in [3].

An extension of the foregoing is presented in [4], in which cross-borehole GPR measurements were used to obtain first-break travel-time picks as input to a joint hydrological-GPR inversion. The forward modelling applied in the MAP (Maximum A Posteriori) inversion procedure was a corrected straight ray approximation. The emphasis of using joint hydrogeological and geophysical inverse modelling is presented in [5]. In this paper the authors consider the use of crosswell GPR data and hydrological data obtained during transient flow events. The inversion proceed iteratively by sequential perturbation of the hydraulic parameters, which indirectly change the dependent geophysical variables (in this case the dielectric constant) until a reasonable match is obtained between predicted and measured geophysical and hydrological data. In contrast to the joint inversion of hydrological and GPR data, [6] inferred temporal variations of moisture content by employing both GPR inversion and resistivity inversion.

More recently, [7] employed crosswell seismic travel-time tomography to obtain the stratigraphy of alluvial deposits by employing data consisting of $\mathrm{P}$ wave arrivals and curved ray tracing forward

\footnotetext{
ae-mail: darylv@ece.ubc.ca
}

This is an Open Access article distributed under the terms of the Creative Commons Attribution-Noncommercial License 3.0, which permits unrestricted use, distribution, and reproduction in any noncommercial medium, provided the original work is properly cited. 
i-DUST 2010

modelling employed in the inversion scheme. An analysis of inversion artifacts, produced by the crosswell geometry, resolution and uncertainty were also presented. Analysis of the resolution of cross-well GPR, described in [8], demonstrated that the inversion smoothed out details but preserved the general character of the hydrological data. A more detailed error analysis was developed in [9]. Data were obtained in the vadose zone at a field site in northern Zealand, Denmark, and correlated errors were accounted for by explicitly including estimated data error covariance matrices in the inversion objective functional.

In our current presentation, we will apply a nonlinear inversion algorithm to crosswell data obtained in the karst aquifer at LSBB (Laboratoire Souterrain à Bas Bruit) near Rustrel France. The first arrival travel-time picks used in the algorithm are obtained via a cross-correlation technique. The first arrival data are then input into an iterative, regularized least square inversion [10]. Modelling of the observed travel-time field is accomplished by the application of a sweeping method [11] that employs an upwinding first-order finite differencing scheme. Ground truth of the inversion is obtained via a qualitative comparison to borehole televiewer images.

\section{TRAVEL-TIME TOMOGRAPHY}

The travel-time inversion is formulated in the typical way as a least-squares minimization of the objective function [10]

$$
\begin{aligned}
\phi(m) & =\phi_{d}+\beta \phi_{m} \\
& =\left\|W_{d}\left(F[m]-d^{o b s}\right)\right\|^{2}+\beta \sum_{n=1}^{N_{r e g}=3} \alpha_{n}\left\|W_{n}\left(m-m_{r e f}\right)\right\|^{2} .
\end{aligned}
$$

The first term, $\phi_{d}$, is the data misfit given by the square of the $l_{2}$ norm of the difference between the predicted data, $d^{\text {pred }}=F[m]$, and the observed data, $d^{o b s} . W_{d}$ is a diagonal matrix with entries equal to the reciprocal of the estimated standard deviation, $1 / \sigma_{i}$, of each measurement. The model parameters are represented by the vector $m$ (multidimensional models are mapped to a vector). The second term is the model norm $\left(\phi_{m}\right)$, which, by appropriate construction of the matrices $W_{n}$, penalizes roughness and the distance from a reference model, $m_{\text {ref }}$.

In this work, the gradient is used as a measure of roughness and is implemented in $W_{1}$ and $W_{2}$ (one for each component of $\left.\nabla\left(m-m_{r e f}\right)\right)$. To measure distance from a reference model, $W_{3}$ is the identity matrix scaled by the square-root of the area of a model cell. Such a weighting reduces the dependency of $\phi_{m}$ on the grid size. The coefficients $\alpha_{n}$ can be adjusted to weight penalization of roughness vs deviation from the reference model (here, $\alpha_{3} \ll \alpha_{1,2}$ due to the lack of an accurate reference model).

The overall inversion is accomplished with a two-loop scheme. The outer loop lowers the regularization parameter, $\beta$, from a large value until the recovered model shows an appropriate trade-off between data fit and model smoothness. Where this happens is somewhat arbitrary, and there are various ways of finding it. If the error associated with the data is known reasonably well ( $W_{d}$ is accurate), then the expected value of $\phi_{d}$ is $N$ (where $N$ is the number of data points). Choosing a value of $\beta$ that produces $\phi_{d} \approx N$ is a reasonable choice, and this is what is used here. For each $\beta$, the inner loop finds a model that minimizes $\phi(m)$.

The forward model, $F[m]$, represents the ray travel-time expression

$$
d_{i}^{\text {pred }}=(F[m])_{i}=\tau\left(\mathbf{r}_{\mathbf{i}}\right)=\int_{L_{i}} s(\mathbf{l}) \mathrm{d} l+T_{0},
$$

where $s(\mathbf{l})$ is the slowness (inverse of velocity) along the ray path and $T_{0}$ is an unknown offset. The time offset is a function of the equipment configuration, and is static for a given measurement setup. It is treated as one additional model parameter (that is not subject to regularization), and must be found 
D. Van Vorst et al.: GPR imaging of the Vaucluse Karst Aquifer using eikonal inversion

during the inversion process. In this algorithm, (2) is never explicitly calculated. Rather, a fast sweeping solver is used to compute an equivalent result using the eikonal equation (Sect. 3).

The forward modelling operation, $F[m]$, is non-linear and therefore cannot be written as a constant matrix multiplying the model parameters (such as can be done for straight-ray tomography). To find the minimum of (1), it is first linearized and then a model update, $\delta m$, that minimizes the linearized problem is found. This process is repeated until the computed gradient of (1) or the model update is sufficiently small. Linearizing (1) about the current model results in

$$
\begin{aligned}
\phi\left(m_{k}+\delta m\right) & =\left\|W_{d}\left(F\left[m_{k}+\delta m\right]-d^{o b s}\right)\right\|^{2}+\beta \sum_{n=1}^{N_{r e g}} \alpha_{n}\left\|W_{n}\left(m_{k}+\delta m-m_{r e f}\right)\right\|^{2} \\
& \approx\left\|W_{d}\left(J_{k} \delta m-\delta d\right)\right\|^{2}+\beta \sum_{n=1}^{N_{r e g}} \alpha_{n}\left\|W_{n}\left(m_{k}+\delta m-m_{r e f}\right)\right\|^{2}
\end{aligned}
$$

where $J_{k}$ are the sensitivities and $\delta d=d^{o b s}-F\left[m_{k}\right]$. The subscript $k$ is the iteration number. The sensitivities are computed using ray tracing (see Sect. 2.1), except for experiments in which bruteforce perturbation was used. Ray tracing produces an approximation of the true sensitivities, and thus negatively affects the evolution of the iterative solution. This negative impact is only slight and is a small price to pay for the vast speed improvement over brute-force perturbation. Ray tracing also produces a sparse sensitivity matrix, which brute-force or other methods (such as adjoint [12]), may not. The sparseness allows for a significant reduction in memory usage and iteration time. Ray tracing is accomplished by following the steepest path backwards from a receiver to the transmitter through the gradient of the travel-time field (Sect 3), in a manner similar to that found in [10].

Setting the gradient of (3) with respect to $\delta m$ to zero results in

$$
\left(J_{k}^{T} W_{d}^{T} W_{d} J_{k}+\beta \sum_{n=1}^{N_{r e g}} \alpha_{n} W_{n}^{T} W_{n}\right) \delta m=J^{T} W_{d}^{T} W_{d} \delta d+\beta \sum_{n=1}^{N_{r e g}} \alpha_{n} W_{n}^{T} W_{n}\left(m_{r e f}-m_{k}\right)
$$

which is a least-squares solution to the over-determined system

$$
\left[\begin{array}{c}
W_{d} J_{k} \\
\sqrt{\beta \alpha_{1}} W_{1} \\
\vdots \\
\sqrt{\beta \alpha_{N}} W_{N}
\end{array}\right] \delta m=\left[\begin{array}{c}
W_{d} \delta d \\
\sqrt{\beta \alpha_{1}} W_{1}\left(m_{r e f}-m_{k}\right) \\
\vdots \\
\sqrt{\beta \alpha_{N}} W_{N}\left(m_{r e f}-m_{k}\right)
\end{array}\right]
$$

Either (4) or (5) can be solved directly for $\delta m$. The lower condition number of the latter should improve convergence if an iterative matrix solver is used.

\subsection{Matrix construction and incorporation $T_{0}$}

To incorporate the unknown travel-time offset, $T_{0}$, of (2) into the inversion procedure, it is treated in an analogous fashion to the other unknown model parameters. The only difference is that it is not subject to any kind of regularization (there is nothing to regularize). To this end, the model parameters, $m$, are 
made to consist of the slowness throughout the model and $T_{0}$, by letting

$$
m=\left[\begin{array}{c}
s_{1} \\
\vdots \\
s_{M} \\
T_{0}
\end{array}\right], \text { and } \delta m=\left[\begin{array}{c}
\delta s_{1} \\
\vdots \\
\delta s_{M} \\
\delta T_{0}
\end{array}\right]
$$

where $M$ is the number of model cells, and $s_{j}$ is the slowness in each cell. The regularization matrices must then be adjusted to remove any attention paid to the last element of the model vector, $m$, by padding them with zeros:

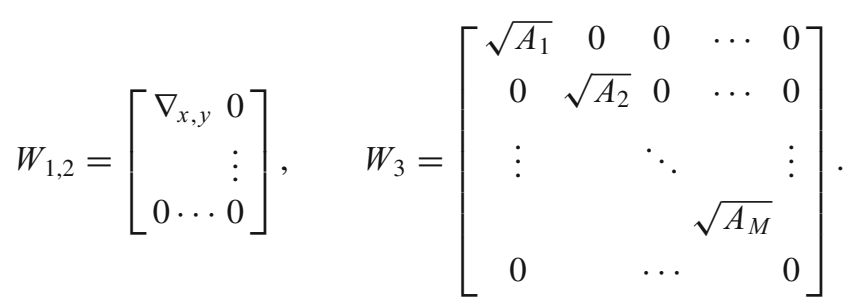

In (7), $A_{i}$ is the area of each model element, and $\nabla_{x, y}$ represents the $x$ or $y$ component of the gradient operator.

Each element of the sensitivity matrix is $J_{i, j}=\partial d_{i}^{\text {pred }} / \partial m_{j}$. The integral in (2) can be expressed as a discrete sum of the product of the length of each ray element with the associated slowness. By realizing that the ray length in a cell through which the ray does not travel is zero, this sum can be extended over all of model space,

$$
d_{i}^{\text {pred }}=\sum_{j=1}^{M} s_{j} l_{i, j}+T_{0},
$$

where $l_{i, j}$ is the length of the ray associated with data point $i$ in model cell $j$. Inspection of (8) clearly shows that $\partial d_{i}^{\text {pred }} / \partial s_{j}=l_{i, j}$ and $\partial d_{i}^{\text {pred }} / \partial T_{0}=1$, provided that the ray path does not change. This allows us to write the sensitivity matrix as

$$
J=\left[\begin{array}{ccccc}
\frac{\partial d_{1}^{\text {pred }}}{\partial s_{1}} & \frac{\partial d_{1}^{\text {pred }}}{\partial s_{2}} & \cdots & \frac{\partial d_{1}^{\text {pred }}}{\partial s_{M}} & 1 \\
\vdots & \vdots & \vdots & \vdots \\
\frac{\partial d_{N}^{\text {pred }}}{\partial s_{1}} & \frac{\partial d_{N}^{\text {pred }}}{\partial s_{2}} & \cdots & \frac{\partial d_{N}^{\text {pred }}}{\partial s_{M}} & 1
\end{array}\right] .
$$

$J$ is a large matrix, but it is very sparse (when computed approximately using rays).

\section{FAST SWEEPING SOLVER}

Travel times for the ray inversion are computed using Zhao's fast sweeping eikonal solver [11], which is related to marching solvers that are used to solve the static Hamilton-Jacobi equation [13, 14]. The solver is extremely simple to implement and accurately handles physical structures with large velocity contrasts. A chief benefit of the solver is that it simply sweeps over the computational domain, rather than requiring a form of sorting to determine the order with which travel times are computed, as is the case in fast marching methods, such as [15]. 
D. Van Vorst et al.: GPR imaging of the Vaucluse Karst Aquifer using eikonal inversion

The fast sweeping algorithm solves the eikonal equation,

$$
|\nabla \tau(\mathbf{r})|=\frac{1}{v(\mathbf{r})}=s(\mathbf{r})
$$

where $s(\vec{r})$ is the position-dependent slowness $(v(\vec{r})$ is velocity) of the medium, and $\tau(\vec{r})$ is the traveltime from source to any location, $\vec{r}$. This is the same travel-time that is given by (2) (with $\left.T_{0}=0\right)$.

Zhao's discretization ${ }^{1}$ of (10) is

$$
\left[\left(\tau_{i, j}-\tau_{x \min }\right)^{+}\right]^{2}+\left[\left(\tau_{i, j}-\tau_{y \min }\right)^{+}\right]^{2}=s_{i, j}^{2} h^{2},
$$

where

$$
(x)^{+}= \begin{cases}x, & x>0 \\ 0, & x \leq 0\end{cases}
$$

$\tau_{x \min }=\min \left(\tau_{i-1, j}, \tau_{i+1, j}\right)$ is and $\tau_{y \min }=\min \left(\tau_{i, j-1}, \tau_{i, j+1}\right)$. The use of $(12), \tau_{x \min }$, and $\tau_{y \min }$ ensure that $\tau_{i, j}$ is computed in a causal fashion. Zhao's algorithm chooses the minimum value of $\tau_{i, j}$ that satisfies (11), and this is given by

$$
\overline{\tau_{i, j}}= \begin{cases}\min \left(\tau_{x \min }, \tau_{y \min }\right)+s_{i, j} h, & \left|\tau_{x \min }-\tau_{y \min }\right| \geq s_{i, j} h, \\ \frac{\tau_{x \min }+\tau_{y \min }+\sqrt{2 s_{i, j}^{2} h^{2}-\left(\tau_{x \min }-\tau_{y \min }\right)^{2}},}{2} & \left|\tau_{x \min }-\tau_{y \min }\right|<s_{i, j} h .\end{cases}
$$

The entire domain is swept through in four alternating directions, and at each grid point (13) is computed. The travel-time at each cell is update to $\tau_{i, j}=\min \left(\overline{\tau_{i, j}}, \tau_{i, j}^{\text {prev }}\right)$, where $\tau_{i, j}^{\text {prev }}$ is the previous travel-time. Four alternating orderings are used to ensure that all possible characteristics (ray directions) are followed. The orderings used here are [11]:

$$
\begin{array}{ll}
\text { (1) } i=1: I, j=1: J, & \text { (2) } i=I: 1, j=1: J, \\
\text { (3) } i=I: 1, j=J: 1, & \text { (4) } i=1: I, j=J: 1 .
\end{array}
$$

It is necessary to use different sweep orders because of the causal nature of the computation. In this work only one set of four sweeps is used to compute the travel-time. Zhao shows that for the distance function (uniform slowness), the results are as accurate as they would be after the algorithm converges. Our recovered velocity model is only slowly varying, and experiments have shown that increasing the number of sweeps does not significantly affect the outcome. In the presence of sharp contrasts, such as those that produce head waves, it would likely be beneficial to increase the number of sweeps.

\section{FIRST-ARRIVAL PICKING}

The objective of first-arrival picking is to accurately determine the time of the first arrival at each receiver location. To avoid hand-picking the data, a simple correlation-based semi-automated picker has been developed for use on the unprocessed raw data. As a starting point, the picker works by finding the cross-correlation peak between a synthesized wavelet (which approximates the transmitted wave), $s_{e s t}(t)$, with the received signal, $s_{r x}(t)$. The highest peak of the normalized cross-correlation,

$$
f(t)=\frac{\int_{-\infty}^{\infty} s_{r x}(\tau) s_{e s t}(t+\tau) \mathrm{d} \tau}{\sigma_{e s t} \sigma_{r x}},
$$

is considered to be the first arrival time, where $\sigma_{e s t}$ and $\sigma_{r x}$ are the standard deviations of the estimated and received signals, respectively.

\footnotetext{
${ }^{1}$ In contrast to previous formulae, the subscripts $i$ and $j$ here represent $x$ and $y$ coordinate indices on a uniform grid, rather than vector or matrix indices.
} 


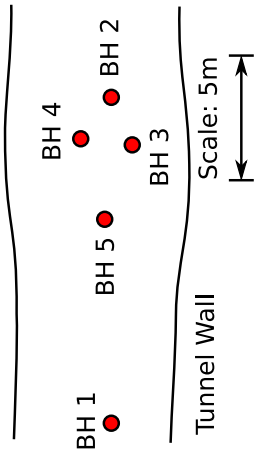

Figure 1. Borehole locations.

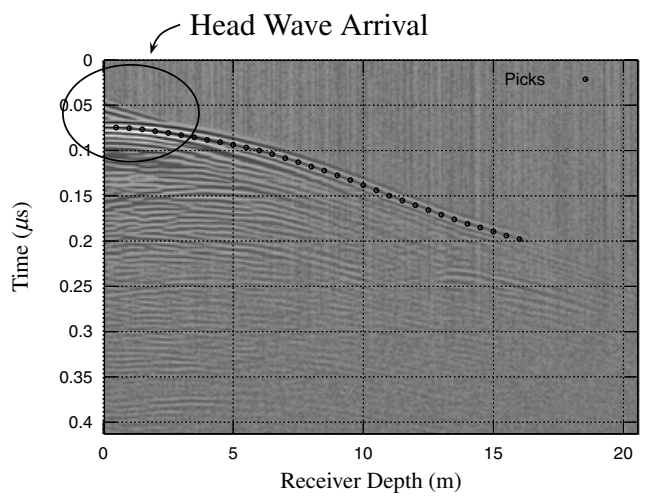

Figure 2. Picking example. The indicated head wave (air wave) arrival is discussed in the text.

The picks are then refined starting with the trace with the highest normalized cross-correlation (or one that is manually specified). The pick for this trace is considered accurate. Traces are re-picked one at a time moving away from the starting trace using the same algorithm, but with a narrow search window for the cross-correlation peak. This has the effect of ensuring that the picked travel-times follow a particular wave crest. The crest that is chosen is inconsequential due to the inclusion of $T_{0}$ in the inversion formulation, as long as the same crest is chosen in each gather. The data set used here has a clearly dominant crest at least somewhere in each gather, which makes cycle skipping over the entire gather an unlikely problem. If there had been some uncertainty, an unknown $T_{0}$ per transmitter location could be added to account for a static offset per gather in an analogous way to how it is added globally in Sect. 2.1.

An example data set collected from the borehole arrangement shown in Fig. 1 is picked in this fashion and is shown in Fig. 2. The figure shows the signal that is received as the receiver is moved from the surface to a depth of $20 \mathrm{~m}$. The transmitter is held stationary near the surface. The plot is exponentially scaled so that weak signals, such as secondary reflections, can be observed. The result of applying the picking algorithm to the data is indicated by the marked points. Figure 2 shows an early and somewhat weaker arrival when both the receive and transmit antennas are near the surface. This is due to propagation of the radar wave through the air, and is intentionally ignored here. By ignoring this early arrival, we can avoid modelling signal propagation through the air above ground and vertically within the boreholes.

\section{RESULTS}

Impulse radar data was collected using two $250 \mathrm{MHz}$ borehole GPR antennas in the borehole arrangement indicated in Fig. 1. Shots were taken by holding the transmit antenna stationary at five equally spaced depths in each $20 \mathrm{~m}$ deep hole, while the receiving antenna was lowered in the borehole directly opposite from the transmitter. Traces were taken at $5 \mathrm{~cm}$ intervals. However, the data was decimated to $25 \mathrm{~cm}$ intervals for use in this work.

The recovered permittivity distribution (determined from the slowness, assuming low loss and magnetic permeability of free space) is shown in Fig. 3, along with borehole televiewer images for holes 1,5 , and 2. Ray density and a few ray paths are shown in Fig. 4 . The velocity inhomogeneity in this case only causes slight ray bending. It is immediately evident that the inversion results superficially match the structure suggested by the viewer images. The light coloured regions at mid-depth in the boreholes correspond to high-porosity regions. If the pores were empty, the effective permittivity of the rock would be lowered. However, the ray inversion results show a higher permittivity, suggesting a higher water content than that of the surrounding rock. 
D. Van Vorst et al.: GPR imaging of the Vaucluse Karst Aquifer using eikonal inversion

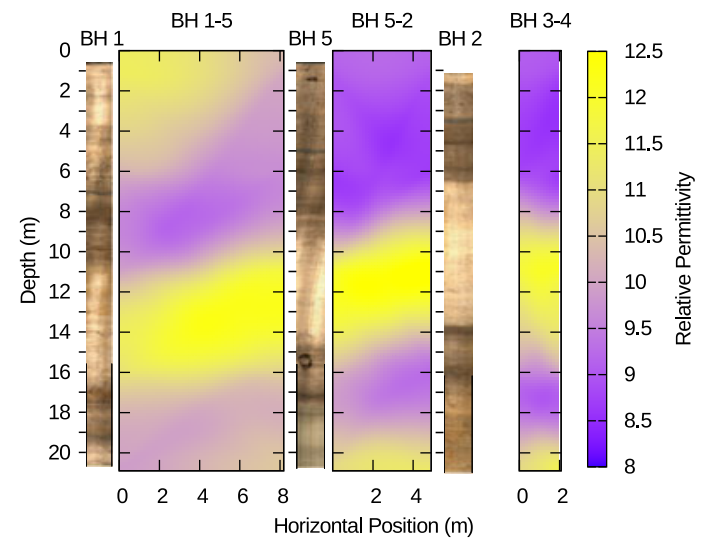

Figure 3. Recovered electric permittivity and borehole televiewer images.

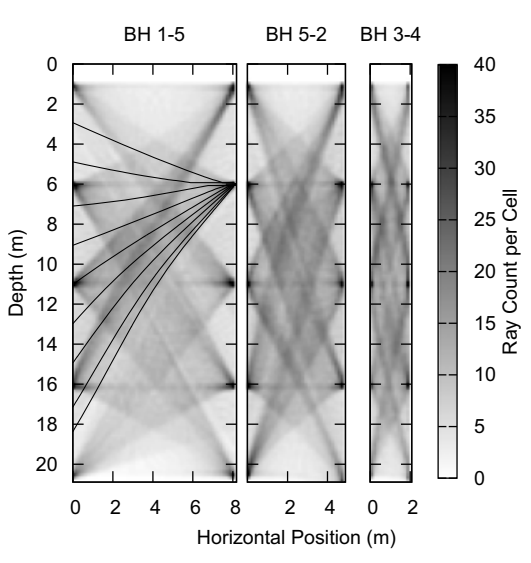

Figure 4. Ray density and example paths.

The foregoing analysis is consistent with the cores pulled from the holes. The cores correspond to a sedimentary facies consisting of low-fractured grainstone with a matrix porosity of $15 \%$ that is intercalated with two highly-fractured wackstone layers with a lower matrix porosity of 5\%. This porosity contrast is consistent with $\mathrm{P}$-wave velocity measurements, with the wackstone layers having an estimated P-wave velocity of $6000 \mathrm{~ms}^{-1}$ and the grainstone layer having a P-wave velocity of $2000 \mathrm{~ms}^{-1}$. The highly-fractured wackstone layers allow water to flow through them, while the lowfractured grainstone layer holds water that is trapped in unconnected pores. Thus, we qualitatively can infer that the grainstone layer has low permeability.

\section{CONCLUSION}

Cross-borehole GPR data was collected at the karst aquifer at LSBB. First arrivals were picked using a cross-correlation technique and inverted for relative permittivity using a regularized least-squares inversion algorithm. The forward modeller used in the inversion was a fast-sweeping eikonal solver which employed Gauss-Seidel relaxation method that does not require any sorting. The inversion results are consistent with televiewer data, measured porosity, and level of fracture of the extracted cores.

These inversion results are currently being used as a starting model for a full waveform inversion algorithm that is in development. The full waveform inversion algorithm and an extension of the traveltime tomography algorithm to $3 \mathrm{D}$ will be published in future works.

The authors would like to thank all the providers of funding and support for this project. Golder Inc. provided in-kind donation for the field work. MALA Geoscience provided two $250 \mathrm{MHz}$ borehole antennas as in-kind support. LSBB and the région PACA provided the infrastructure. Direct funds for travel and perdiem were provided by the project ANR MAXWELL, while the funding to drill and log the boreholes was provided by the project ANR HPPPCO2.

\section{References}

[1] S. Hubbard, Y. Rubin, E. Majer, Water Resour. Res. 35(6), 1809 (1999)

[2] S. Hubbard, Y. Rubin, J. Contam. Hydrol. 45(1-2), 3 (2000)

[3] J. Huisman, S. Hubbard, J. Redman, A. Annan, Vadose Zone J. 2(4), 476 (2003)

[4] M. Kowalsky, S. Finsterle, J. Peterson, S. Hubbard, Y. Rubin, E. Majer, A. Ward, G. Gee, LBNL internal publication (2005) 


\section{i-DUST 2010}

[5] N. Linde, J. Chen, M. Kowalsky, S. Hubbard, NATO SCI S SS IV EAR, pp. 9-44 (2006)

[6] A. Binley, P. Winship, L. West, M. Pokar, R. Middleton, J. Hyd. 267(3-4), 160 (2002)

[7] G. Moret, M. Knoll, W. Barrash, W. Clement, Geop. 71 B63 (2006)

[8] J. Brainard, G. Hammond, D. Alumbaugh, D. La Brecque, Tech. rep., Sandia National Laboratories (2007)

[9] K. Cordua, M. Looms, L. Nielsen, Vadose Zone J. 7(1), 263 (2008)

[10] D. Aldridge, D. Oldenburg, J. Seism. Explor. 2, 257 (1993)

[11] H. Zhao, Math. Comput. 74(250), 603 (2004)

[12] S. Leung, J. Qian, Commun. Math. Sci. 4(1), 249 (2006)

[13] S. Jin, M.A. Katsoulakis, J. Differ. Equations 138(2), 380 (1997)

[14] C.Y. Kao, S. Osher, J. Qian, J. Comput. Phys. 196(1), 367 (2004)

[15] J. Vidale, B. Seismol Soc. Am. 78(6), 2062 (1988) 